\title{
Otolaryngology, Hearing, Knowledge and Globalization
}

\author{
Jorge Francisco Moises Hernandez* \\ Service of Otolaryngology and Head and Neck Surgery, General Hospital of Mexico, Mexico
}

*Corresponding author: Jorge F Moisés H, Service of Otolaryngology and Head and Neck Surgery, General Hospital of Mexico, Mexico.

Received Date: October 08, 2018

Published Date: October 30, 2018

\section{Editorial}

Otorhinolaryngology as a specialty fulfills the important function of preserving among many other functions, hearing and language, essential elements for the individual as a fundamental part of social and familiar interaction that will give a huge range of knowledge and experiences, enabling human growth and development that will transform him into a full human being in the individual and productive to society. This individual growth is largely due to the hearing process that allows the acquisition of language which in turn will be the basis of the transmission of knowledge and therefore of the preservation of culture and civilizations.

In the last decade and as a direct consequence in the most recent advances in technology, mainly in lighting and photography, Otorhinolaryngology as a discipline has had an impressive advance allowing us to understand in a clearer and more precise way anatomical and physiological aspects that we did not know or thought we knew. Making the pathological processes clearer and therefore the therapeutic. On the other hand, the science general advance occurs within the framework of consensus in the different forums that exist for this purpose. Including of course the printed and electronic written media in which the different authors can capture their advance in knowledge, their experiences and the cases that have them some particular teaching. Thus, the communication of knowledge by the written language that is acquired through hearing, converge in these works allowing us to be more complete every day and reach more doctors around the world.

Listening, language, writing is knowledge that converge with scientific and technological advances in which our specialty is active participant in a globalized world!

To the parallel of what was described above, the great advances made by communication technologies and the globalizing event that they have created around the world. There are no longer isolated medical practices by countries or region. The world is one and in this global village the knowledge generated in Europe, America or Asia can be immediately disseminated, corroborated, discarded or enriched. As is the case with this important online scientific publication.

\section{Acknowledgement}

Today I am honored to be able to address all the doctor in the world with these simple words in the inaugural issue of this magazine, which will be undoubtedly the most effective and reliable knowledge diffusion vehicle that our colleagues from all over the world can count on. To translate and review its contribution to the knowledge and advancement of medical sciences. Favoring the diffusion of knowledge to all corners of our planet. Making knowledge the tool that will contribute to the growth of our specialty and improving the quality of care we provide to our patients. 